\title{
Wavefront control architecture and expected performance for the TMT Planetary Systems Imager
}

Olivier Guyon, Benjamin Mazin, Michael Fitzgerald, Dimitri Mawet, Christian Marois, et al.

Olivier Guyon, Benjamin Mazin, Michael Fitzgerald, Dimitri Mawet, Christian Marois, Andy Skemer, Julien Lozi, Jared Males, "Wavefront control architecture and expected performance for the TMT Planetary Systems Imager," Proc. SPIE 10703, Adaptive Optics Systems VI, $107030 Z$ (17 July 2018); doi: $10.1117 / 12.2314331$

Event: SPIE Astronomical Telescopes + Instrumentation, 2018, Austin, Texas, United States 


\title{
Wavefront Control Architecture and Expected Performance for the TMT Planetary Systems Imager
}

\author{
Olivier Guyon ${ }^{a, b, c}$, Benjamin Mazin ${ }^{d}$, Michael Fitzgerald ${ }^{e}$, Dimitri Mawet ${ }^{f}$, Christian Marois ${ }^{g}$, \\ Andy Skemer ${ }^{h}$, Julien Lozi ${ }^{c}$, Jared Males ${ }^{b}$, TMT-PSI instrument team \\ ${ }^{a}$ Astrobiology Center; National Institutes of Natural Sciences, Tokyo, JAPAN \\ ${ }^{b}$ Steward Observatory, University of Arizona, Tucson, AZ 85721, USA \\ ${ }^{c}$ National Astronomical Observatory of Japan, Subaru Telescope, National Institutes of \\ Natural Sciences, Hilo, HI 96720, USA \\ ${ }^{d}$ University of California Santa Barbara, USA \\ eUniversity of California Los Angeles, USA \\ ${ }^{\text {Caltech, USA }}$ \\ ${ }^{g}$ National Research Council Canada, CANADA \\ ${ }^{h}$ University of California Santa Cruz, USA
}

\begin{abstract}
The Planetary Systems Imager (PSI) is a modular instrument optimized for direct imaging and characterization of exoplanet and disks with the Thirty Meter Telescope (TMT). PSI will operate across a wide wavelength range $(\approx 0.6-5 \mu \mathrm{m})$ to image exoplanets and circumstellar disks in both reflected light and thermal emission. Thanks to the TMT's large collecting area, PSI will have the sensitivity to directly image and spectrally characterize large gaseous planets with unprecedented sensitivity. PSI will also be capable of imaging rocky planets in the habitable zones of the nearest M-type stars in reflected light and search for biomarkers in their atmospheres.

Imaging habitable planets in reflected light is PSI's most challenging goal, requiring high contrast imaging (HCI) capabilities well beyond what current instruments achieve. This science goal drives PSI's wavefront sensing and control requirements and defines the corresponding architecture discussed in this paper. We show that PSI must deliver $1 \mathrm{e}-5$ image contrast at $\approx 15$ mas separation at $\lambda \approx 1 \mu m-1.5 \mu m$, and that a conventional extreme-AO architecture relying on a single high speed wavefront sensor (WFS) is not sufficient to meet this requirement. We propose a wavefront control architecture relying on both visible light $(\lambda<1.1 \mu \mathrm{m})$ sensing to optimize sensitivity, and near-IR $(\lambda>1.1 \mu \mathrm{m})$ sensors to address wavefront chromaticity terms and provide high contrast imaging capability. We show that this combination will enable speckle halo suppression at the $<$ 1e-5 raw contrast level in near-IR, allowing detection and spectroscopic characterization of potentially habitable exoplanets orbiting nearby M-type stars.
\end{abstract}

Keywords: Exoplanets, High Contrast Imaging, Adaptive Optics, Coronagraphy

\section{INTRODUCTION TO TMT-PSI}

\subsection{Scientific background}

Several thousand exoplanets have been identified using indirect detection techniques (radial velocity and transit). While most are giant gas planets due to detection biases, smaller rocky planets have been identified in recent years. There is now solid statistical evidence that rocky planets between $\approx 0.5$ and $\approx 5$ Earth masses orbiting within their star's habitable zone are a frequent occurrence. Such planets have the potential to sustain liquid water and biological activity; they are referred to as potentially habitable.

Further author information: (Send correspondence to O.G.)

O.G.: E-mail: oliv.guyon@gmail.com, Telephone: 18182938826

Adaptive Optics Systems VI, edited by Laird M. Close, Laura Schreiber,

Dirk Schmidt, Proc. of SPIE Vol. 10703, 107030Z · @ 2018 SPIE

CCC code: $0277-786 \mathrm{X} / 18 / \$ 18 \cdot$ doi: $10.1117 / 12.2314331$

Proc. of SPIE Vol. 10703 107030Z-1 


\subsection{TMT-PSI observations}

Direct imaging with high contrast cameras/instruments is fundamentally well-suited to characterize exoplanetary systems. Multiple planets can be identified, and their orbits measured. Circumstellar disks can also be mapped, and planets/disk interactions measured. As planet light is isolated from the considerably brighter starlight, high SNR photometry, spectro-photometry, polarimetry and spectroscopy become possible. These are the key goals of the Thirty Meter Telescope Planetary Systems Imager (TMT-PSI) instrument, ${ }^{1-3}$ which is designed to perform the following observations:

- Thermal emission from exoplanets. TMT-PSI will take advantage of the telescope's large collecting area and small angular resolution to observe the thermal emission from young planets with unprecedented sensitivity. The current small sample of directly imaged planets will be significantly increased, and the instrument will perform detailed characterization of young planets ranging in mass from Neptune to the planet / brown dwarf limit.

- Circumstellar disks will be mapped in visible and near-IR light. Direct imaging will be augmented by polarimetric imaging to both enhance detection limits and constrain dust properties.

- Reflected light exoplanet imaging will be possible thanks to high contrast imaging capability and small inner working angle. This will allow detection and characterization of nearby mature planets within habitable zones.

TMT-PSI will additionally observe non-exoplanet targets for scientific investigations uniquely enabled by its angular resolution and sensitivity. These include stellar surfaces (spectro-imaging), stellar multiplicity, compact objects (accreting binaries, black holes and neutron stars), and active galactic nuclei.

\subsection{Instrument Architecture: Overview}

Most of TMT-PSI observations longward of $\approx 2 \mu \mathrm{m}$ will be limited by thermal background and do not critically depend on aggressive ExAO performance, allowing conservative, well-understood approaches to instrument design.

In contrast, visible and near-IR observations will be limited by residual wavefront errors, requiring high-performance coronagraphic technologies and advanced wavefront sensing/control techniques. Among TMTPSI's observations, direct imaging and spectroscopic characterization of nearby habitable planets in reflected light is the most challenging and will drive the optical/near-IR instrument design. High contrast imaging performance will be enabled by extreme adaptive optics (ExAO) and small inner working coronagraphy. The reflected light observations will be best performed in visible and near-IR $(0.6 \mu m<\lambda<2 \mu m)$, where the coronagraphic inner working angle can resolve the habitable zones of nearby M-type stars and therefore allow direct imaging of moderate-contrast habitable exoplanets.

TMT-PSI will provide both facility-class, reliable long wavelength observation capabilities, and deploy cuttingedge technologies to meet the challenging high contrast performance required for reflected light exoplanet observations. This will be achieved by deploying two instrument modules:

- PSI-red will perform adaptive optics correction for the whole instrument to deliver high imaging performance longward of $2 \mu \mathrm{m}$ for imaging and spectrocopy. The common AO hardware and cryogenic instruments will be built as facility-class modules optimized for reliability, stability and performance in the background-limited regime.

- PSI-blue will perform additional ExAO correction and high contrast imaging for imaging, spectroscopy and polarimetric imaging in the $0.6 \mu m-2 \mu m$ range. PSI-blue will employ advanced technologies and will be upgradeable to take advantage of new hardware capabilities (detectors, coronagraph masks, computer equipment) critical to the instrument's high contrast performance. 


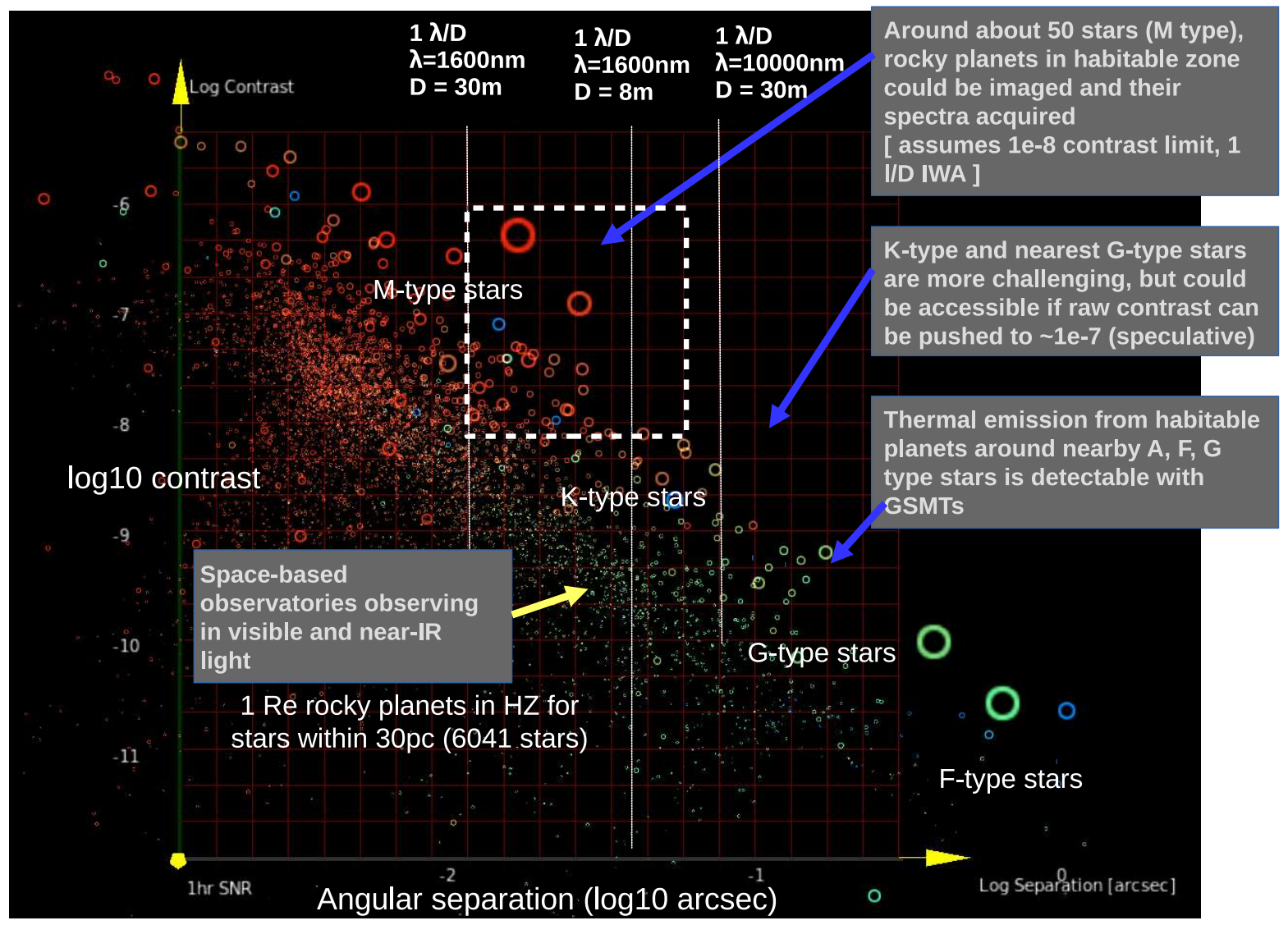

Figure 1. TMT PSI potentially habitable planets sample. This figure indicates the location, in angular separation (x-axis) and contrast (y-axis) of hypothetical Earth analogs around each star within 30pc. Habitable planets orbiting sun-like stars cannot be detected in reflected light from ground telescopes due to extreme contrast levels $(\approx 1 \mathrm{e}-10)$, but they are accessible with TMT around M-type stars thanks to more moderate contrasts.

\section{HABITABLE EXOPLANET SAMPLE AND REQUIRED RAW CONTRAST}

\subsection{The Reflected Light Habitable Exoplanets Sample}

PSI's most challenging goal is to image and characterize habitable planets orbiting nearby stars. We focus in this paper on the reflected light observations, where starlight reflected by the planet's atmosphere and/or surface is captured by imaging camera(s) and spectrograph(s). These challenging observations will drive PSI's wavefront sensing and control architecture. Another path to imaging habitable planets with large telescopes, which is not discussed in this paper, is to capture their thermal emission ${ }^{4}$ in the 5 to $13 \mu \mathrm{m}$ spectral range where the blackbody emission of a temperate planet peaks.

The main fundamental challenges to reflected light exoplanet imaging are the contrast level between the planet and the star, the small angular separation between the two bodies, and the low intrinsic brightness of the planet. Figure 1 shows the location of hytotherical habitable planets (one planet per star) in the angular separation vs. contrast plane. Habitable planets at large angular separations $(>\approx 0.1$ arcsec $)$ are $\approx 1 \mathrm{e} 10 \times$ fainter than the stars they orbit and can only be imaged from space. While large ground-based telescopes cannot provide access to extremely high contrast levels due to residual atmospheric turbulence, they do offer 10mas-level angular resolution, so they can access planets at more moderate contrast level in the left part of the figure.

Habitable planets orbiting nearby M-type stars appear to be particularly well-suited for direct imaging observations with large ground-based telescopes: the contrast is relatively moderate $(\approx 1 \mathrm{e}-7)$ and potentially within 
reach of an extreme adaptive optics (ExAO) system; the angular separation can be resolved thanks to the large telescope aperture, and the telescope's collecting area allows for spectrocospic observations of the planets.

\subsection{Exoplanet Detection Process}

TMT-PSI relies on three core techniques to image and characterize exoplanets:

- Coronagraphy is deployed to optically suppress starlight while maintaining high exoplanet throughput.

- Wavefront sensing and control mitigates atmospheric turbulence and ensures that the residual starlight speckle halo brightness is sufficiently faint.

- Differential detection techniques are employed to identify exoplanet light among the residual starlight halo.

Starlight suppression systems have reached raw contrast levels below 1e-8 at optical wavelengths in laboratory environments. Coronagraphs have also recently been successfully designed for centrally obscured apertures with thick spiders, demonstrating that static diffraction from TMT's aperture can fundamentally be optically suppressed to the $\approx 1 \mathrm{e}-8$ raw contrast level representative of TMT-PSI's challenging reflected light exoplanets.

Achieving these raw contrast levels requires nm-level wavefront control, which is approximately two orders of magnitude beyond what current operating ExAO systems routinely deliver on 8-m class telescopes. Raw contrast will therefore be limited by the wavefront sensing and control performance, and the corresponding speckle halo surface brightness will be significantly brighter than habitable zone reflected light exoplanets.

Differential detection techniques must therefore be employed to identify planet light among the comparatively brighter speckle halo. Current ExAO systems rely on Angular and Spectral Differential Imaging (ADI, SDI) to detect sources approximately $100 \times$ fainter than the speckle halo raw contrast. Thanks to recent advances in detectors and algorithms, TMT-PSI will additionally benefit from coherence-based differential detection approaches and high spectral resolution differential detection. Wavefront sensing telemetry can also be used to estimate realtime focal plane residual light to further augment detection sensitivity. Differential detection approaches are an active research area offering significant benefits for exoplanet imaging with TMT-PSI.

\subsection{Optimal Observation Wavelength for Reflected Light Habitable Exoplanets Imaging}

We explore in this section what is the optimal wavelength choice for reflected light imaging of habitable exoplanets with TMT. We assume here that exoplanet detections are limited by photon noise from the residual starlight halo, and we assume that the raw contrast is equal to $1 \mathrm{e}-6$ at $\lambda=1 \mu m$ (this arbitrary choice will later be revisited).

We now consider that the observing wavelength is a free parameter, and count the number of stars for which an Earth analog, if present, could be detected and characterized. The detection threshold is set to SNR=10 for acquisition of a $\mathrm{R}=40$ spectrum within $1 \mathrm{hr}$ exposure time. As wavelength increases, the raw contrast improves as $\propto \lambda^{-2}$, the photon flux from the planet and the star varies according to spectral type, and the coronagraph inner working angle (set to $1.3 \lambda / D$ ) increases.

Results, presented in Figure 2, show that the number of observable exoplanets peaks around $\mathrm{J}$ and $\mathrm{H}$ band. The sample size is reduced by poor contrast at shorter wavelength, and by larger IWA at longer wavelength. For the assumed raw contrast value $(1 \mathrm{e}-6$ at $1 \mu \mathrm{m})$, habitable planets are observable around a few dozen stars. The sample size is also given for the unrealistic "no starlight" (perfect wavefront) case: numbers are considerably higher (hundreds), demonstrating that further raw contrast improvements would significantly grow sample size.

Figure 2 also shows that the best targets are M-type stars, and that, as wavelength increases, slightly hotter stars are favored,

The optimal wavelength range for imaging and spectroscopic characterization of potentially habitable planets is near-IR: $\mathbf{J}$ band and $\mathrm{H}$ band $(\approx 1 \mu \mathrm{m}$ to $1.8 \mu \mathrm{m})$. The most favorable targets are mid-M type main sequence stars. 


\section{I band: 24 planets}

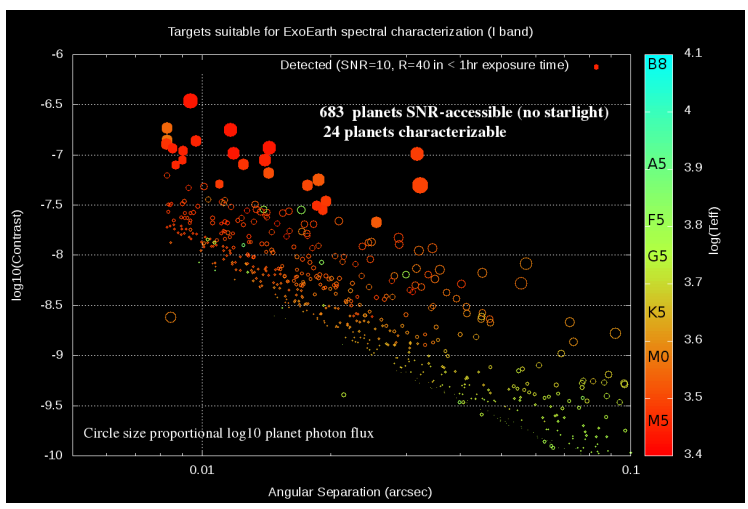

$\mathrm{H}$ band: 38 planets

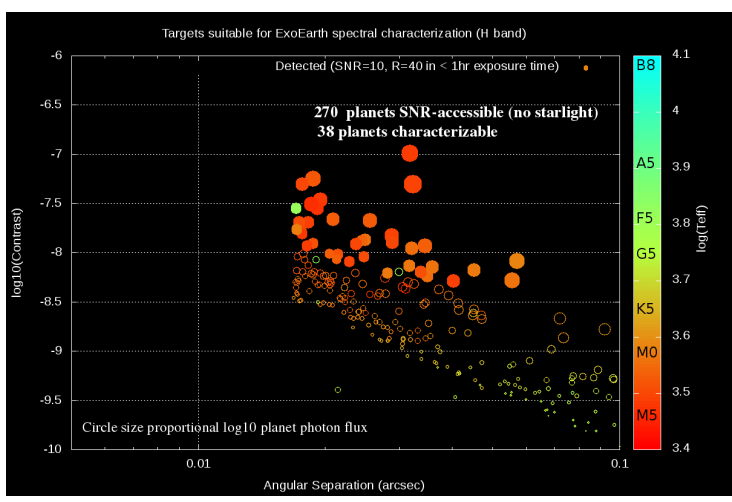

J band: 46 planets

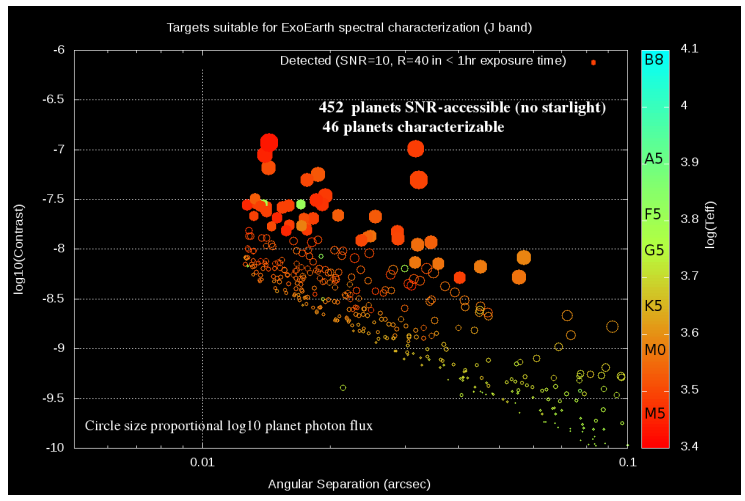

K band: 28 planets

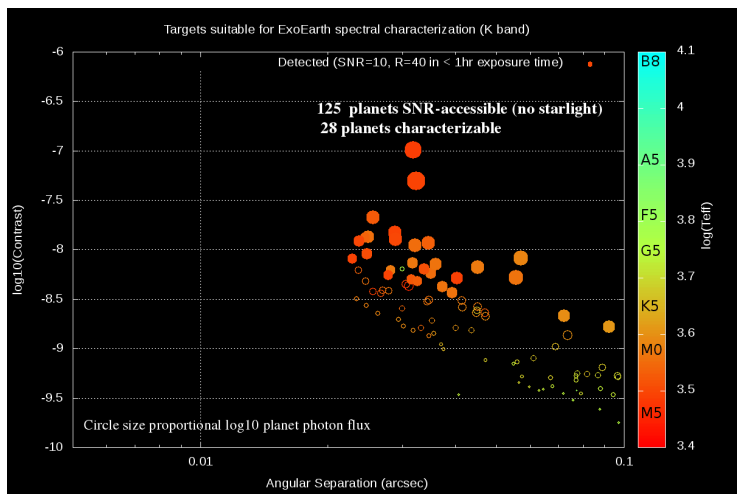

Figure 2. Stars around which Earth-like planets are accessible, as a function of wavelength in the photon-noise limited regime.

\subsection{Raw Contrast Requirement}

TMT-PSI's raw contrast requirement is more carefully derived here assuming that detection is limited by the speckle halo photon noise, assuming that spectroscopic characterization requires a $R=100$ spectrum to be obtained at $S N R=10$ in $1 \mathrm{hr}$ of exposure time.

We consider PSI's observation of the Ross $128 \mathrm{~b}$ planet as representative of characterization of a nearby potential habitable exoplanets. Ross 128 is a M4 main sequence star at $3.34 \mathrm{pc}$, with apparent magnitudes $m_{V}=11.153, m_{I}=8.184, m_{J}=6.505$ and $m_{H}=5.945$. Ross $128 \mathrm{~b}$, identified by radial velocity, orbits the star at a $0.0496 \mathrm{AU}$ or $15 \mathrm{mas}$ semi-major axis, corresponding to $1.33 \lambda / D$ in $\mathrm{H}$ band $\left(\lambda_{c}=1.63 \mu \mathrm{m}\right)$ and $1.77 \lambda / D$ in $\mathrm{J}$ band $\left(\lambda_{c}=1.22 \mu \mathrm{m}\right)$ for TMT's $30 \mathrm{~m}$ aperture. The planet is assumed to have an Earth albedo, and a 1.1 $R_{\text {Earth }}$ radius. The resulting reflected light contrast is $\mathrm{C}=7.4 \mathrm{e}-8$.

We assume a $10 \%$ overall photometric efficiency from the top of the atmosphere to the photo-electron on the spetrometer's noise-free detector. Over each $1 \%$ wide spectral bin, the instrument collects 2.5 ph.s ${ }^{-1}$ and 2.1 ph.s $s^{-1}$ from the planet in respectively $\mathrm{J}$ and $\mathrm{H}$ band. The corresponding allowable raw contrast levels to meet the science requirement are 1.0e-5 in $\mathrm{J}$ band and $1.5 \mathrm{e}-5 \mathrm{in} \mathrm{H}$ band.

In this photon noise limited case, the planet flux is $135 \times(\mathrm{J}$ band $)$ and $90 \times(\mathrm{H}$ band $)$ fainter than the residual starlight speckle halo, so the differential detection technique(s) employed to separate planet from starlight must operate at the $<1 \%$ noise level. Additionally, the coronagraph system must be able to deliver $<1 \mathrm{e}-5$ raw contrast in the absence of wavefront errors, but taking into account the stellar angular diameter. While quantitative analysis of these requirements is outside of the scope of this paper, we note that they appear chal- 
lenging yet reachable based on recent coronagraphic laboratory results and achieved on-sky differential detection / postprocessing performance.

To characterize nearby potentially habitable planets around M-type stars, TMT PSI will need to deliver $\approx 1 \mathrm{e}-5$ raw contrast in J-band.

\section{WAVEFRONT SENSING AND CONTROL FUNDAMENTALS}

In this section, we first consider a conventional $\mathrm{AO}$ system architecture, with a single ExAO wavefront sensor operating at the most sensitive wavelength, and driving a single deformable mirror. The analysis seeks to identify error terms that TMT-PSI WFS/C must address to meet the 1e-5 raw contrast requirement derived in $\S 2.4$.

\subsection{Primary Wavefront Sensing Wavelength}

The primary WFS must operate at or near the wavelength offering the best measurement sensitivity. The wavelength choice is a tradeoff between photon flux (which favors near-IR for M-type stars) and single photon information content (which favors short wavelengths). In the small aberration linear regime considered here, the wavefront sensor measurement signal amplitude for a given wavefront aberration is inverse proportional to wavelength due to the path length to phase scaling: Wavefront sensing at shorter wavelength will produce a stronger signal. Measurement noise is also proportional to the inverse square root of the available number of photon $F$. For a given source spectral type, the optimal sensing wavelength can therefore be derived by maximizing $\sqrt{F} / \lambda$.

Table 1. Optimal Wavefront Sensing Wavelength - Linear Regime

\begin{tabular}{l|c|c|c|c|c|c}
\hline $\begin{array}{l}\text { Spectral } \\
\text { Type }\end{array}$ & $\begin{array}{c}\text { Teff } \\
{[\mathrm{K}]}\end{array}$ & Optimal $_{\text {Band }^{\mathrm{a}}}$ & Photon flux $^{\mathrm{b}}$ & \multicolumn{3}{|c}{ Flux gain relative to $\ldots$} \\
\hline B0V & 31500 & $\mathrm{U}$ & $1.08 \mathrm{e} 10$ & 2.14 & 12.06 & 1337.0 \\
A0V & 9700 & $\mathrm{~B}$ & $5.01 \mathrm{e} 7$ & 1.00 & 4.25 & 204.7 \\
F0V & 7200 & $\mathrm{~B}$ & $1.05 \mathrm{e} 7$ & 1.00 & 2.78 & 82.1 \\
G0V & 5920 & $\mathrm{~B}$ & $1.34 \mathrm{e} 6$ & 1.00 & 1.80 & 33.7 \\
K0V & 5280 & $\mathrm{~B}$ & $3.26 \mathrm{e} 5$ & 1.00 & 1.33 & 17.6 \\
M0V & 3850 & $\mathrm{R}$ & $3.53 \mathrm{e} 4$ & 2.03 & 1.00 & 3.93 \\
M4V & $\mathbf{3 2 0 0}$ & I & $\mathbf{4 . 6 5 e} 3$ & $\mathbf{1 2 . 5}$ & $\mathbf{1 . 8 0}$ & $\mathbf{2 . 8 3}$ \\
M8V & 2500 & $\mathrm{~J}$ & $6.00 \mathrm{e} 2$ & 150.0 & 11.6 & 1.98 \\
\hline
\end{tabular}

Table 1 lists the optimal wavefront sensing band, assuming a fixed relative spectral bandwidth $d \lambda / \lambda$. Photon fluxes are listed for a $10 \%$ effective spectral band at the optimal sensing wavelength, for main sequence stars at 10pc. The optimal WFS band is a function of stellar type, and is I band for mid-M spectral types. Sensing in R-band or H-band are less ideal options, and would be approximately half as efficient.

The optimal primary WFS wavelength for reflected light imaging of habitable planets is I band $(\approx 0.9 \mu \mathrm{m})$.

\subsection{Raw Contrast Error Budget}

We use analytical methods ${ }^{5}$ to establish a raw contrast error budget, shown in Figure 3 for the Ross 128 observation adopted as a representative example. The simulation only contains error terms due to residual wavefront errors, and does not include the contributions of coronagraph optics imperfections, stellar angular size, or sky background.

Wavefront sensing is performed in I band to maximize sensitivity, as described in $\S 2.3$. The effective WFS bandwidth (product of spectral bandwidth and efficiency) is 108nm. The WFS is a non-modulated Pyramid WFS, 
Raw Contrast Terms in ExAO High Contrast Imaging

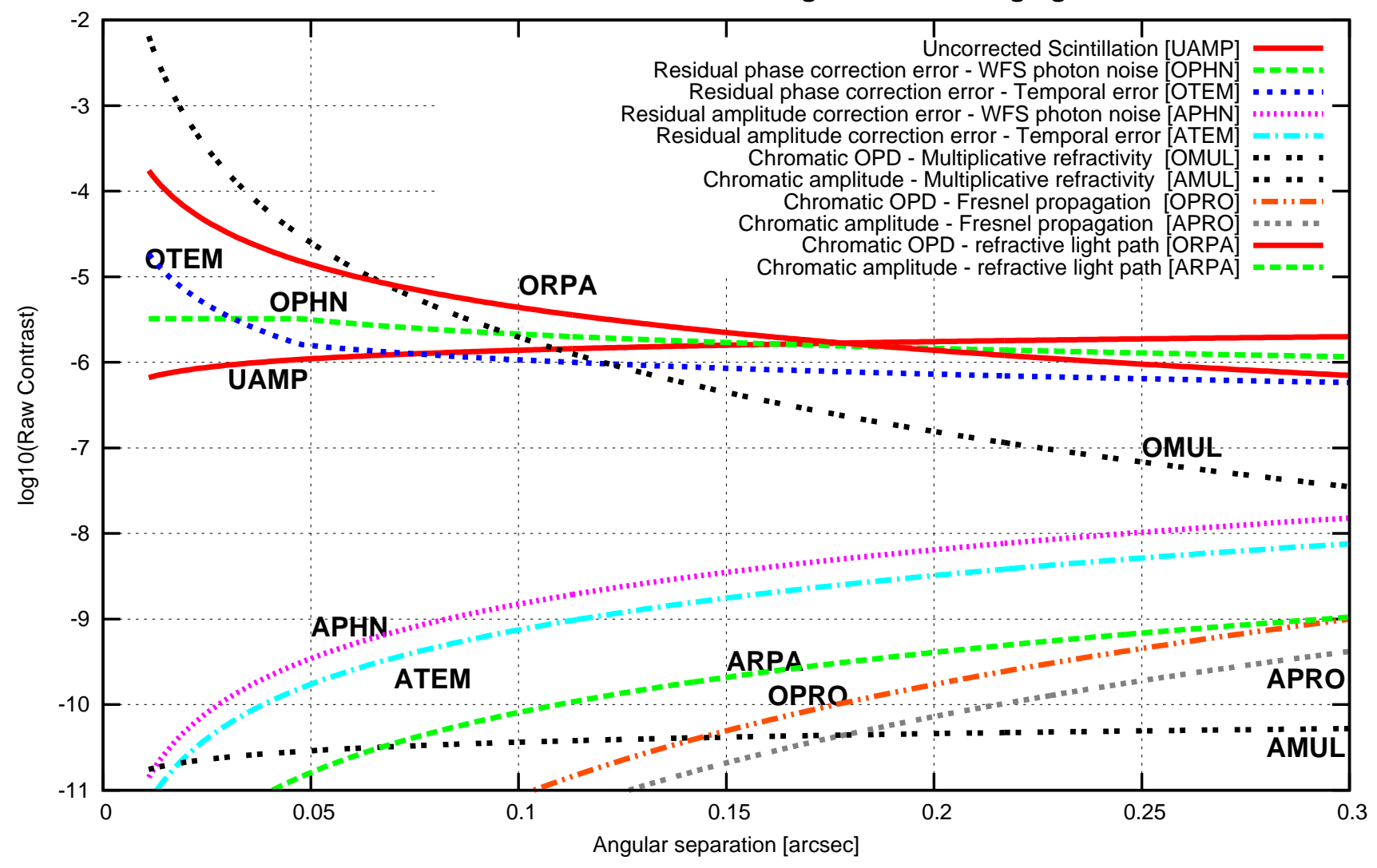

Figure 3. Raw contrast terms for a simulated observation of star Ross 128. Wavefront sensing is performed in I band, and raw contrast is measured in $\mathrm{J}$ band. Curves start at a $1.3 \lambda / D$ angular separation corresponding to the coronagraph inner working angle (IWA). The region of interest for habitable planet imaging is within 50mas, at the leftmost part of this figure.

operating at the diffraction-limited photon noise sensitivity: this choice offers significantly better sensitivity than a seeing-limited WFS such as the Shack-Hartmann WFS.

The AO control law is simple integrator loop, for which the frame rate and gain are optimally adjusted, at each spatial frequency, to minimize the sum of the WFS photon noise and temporal error raw contrast terms (OPHN and OTEM terms).

This analysis reveals that the dominant error terms contribute to a $\approx 1 \mathrm{e}-3$ raw contrast level within 50 mas of the optical axis, which is $100 \times$ larger than the $1 \mathrm{e}-5$ required raw contrast. The error terms that contribute to 1e-6 raw contrast or more at small angular separation, are:

- Chromatic OPD due to multiplicative refractivity [OMUL]. The refractive index of air is chromatic, so there is multiplicative factor in optical path length (OPD) between the WFS wavelength and the high contrast imaging wavelength. This term is the largest source of residual starlight near the optical axis, at about 1e-3 contrast level.

- Chromatic OPD due to refractive light path [ORPA]. The light path from the target to the telescope is chromatic due to atmospheric refraction, creating a chromatic shear term between the WFS and imaging wavelengths. This term is computed here for a $40 \mathrm{deg}$ zenith angle pointing atop Maunakea, and is about 1e-4 contrast level at the coronagraph's IWA. 
- WFS Photon noise and time lag [OPHN and OTEM]. These two terms are fundamentally linked, and their amplitude is driven by the incoming starlight flux and control loop speed. The ExAO WFS frame rate and gain are optimally chosen to minimize the sum of the two terms.

- Scintillation [UAMP]. As light propagates through the turbulent atmosphere, phase errors are partially converted into amplitude modulation. This effect is at the 1e-6 raw contrast level, so addressing it is optional.

All other error terms evaluated in Figure 3 are below 1e-9 raw contrast near the optical axis, so they do not need to be addressed.

A conventional ExAO WFS/C architecture does not meet TMT-PSI's 1e-5 raw contrast requirement. In addition to deploying a fast ExAO primary WFS/C control loop, TMTPSI WFS/C architecture must reduce wavefront OPD chromaticity residuals by $100 \mathrm{x}$, and must improve the WFS noise / time lag residual by a factor of a few. If possible, scintillation should also be mitigated.

\section{WAVEFRONT SENSING AND CONTROL ARCHITECTURE}

\subsection{Sensors and Control Loops}

To address limitations identified in $\S 3$, the TMT-PSI WFS/C architecture must include both visible (I band) and near-IR (at or near the science imaging wavelength) wavefront sensing. Additionally, non-common path wavefront errors must be addressed to reach and maintain high contrast, and wavefront sensors must be capable of reliably measuring the residual aberrations in the high contrast image for post-processing.

Table 2. TMT-PSI Wavefront Sensors.

\begin{tabular}{|l|c|c|c|r|}
\hline WFS & Wavelength & Speed & Number of modes & Function \\
\hline Near-IR common WFS & $1-5 \mu \mathrm{m}$ & $1-2 \mathrm{kHz}$ & $\approx 10,000$ & Provides near-IR high SR PSF \\
Visible ExAO WFS & $0.6-1.1 \mu \mathrm{m}$ & $1-5 \mathrm{kHz}$ & $2,000-10,000$ & High contrast halo suppression \\
Coronagraphic LOWFS & $1.2-1.8 \mu \mathrm{m}$ & $2-5 \mathrm{kHz}$ & $\approx 20$ & Coronagraph alignment \\
Focal plane speckle WFS & $1.2-1.8 \mu \mathrm{m}$ & $0.5-1 \mathrm{kHz}$ & $\approx 1000$ & Speckle sensing + Science \\
\hline
\end{tabular}

These requirements are addressed in PSI by deploying 4 wavefront sensors, listed in Table 2. The sensors and associated control paths are also shown in the instrument block diagram in Figure 4:

- LOOP [1]: A near-IR common WFS provides the first correction stage, reducing the large incoming OPD errors to deliver a well-corrected near-IR PSF for PSI-blue. A dedicated DM, common to the whole instrument, performs the correction. This initial AO loop can operate independently of PSI-blue.

- LOOP [2]: Thanks to the initial AO correction loop, a visible ExAO WFS located inside PSI-blue can operate at the telescope's diffraction limit, providing maximum sensitivity for measurement of small residual wavefront errors. This WFS drives PSI-blue's high speed tweeter DM.

- LOOP [3]: A coronagraphic low-order WFS captures near-IR starlight that is rejected by the coronagraph to lock the fine alignment that is critical to starlight suppression. The sensor measures tip-tilt and a few other low-order modes.

- LOOP [4]: A speckle control loop operates from focal plane image(s) to track and suppress speckles in real time. The loop ensures that no static or slow speckle is left in the image, and provides absolute measurement of residual starlight for post-processing. 


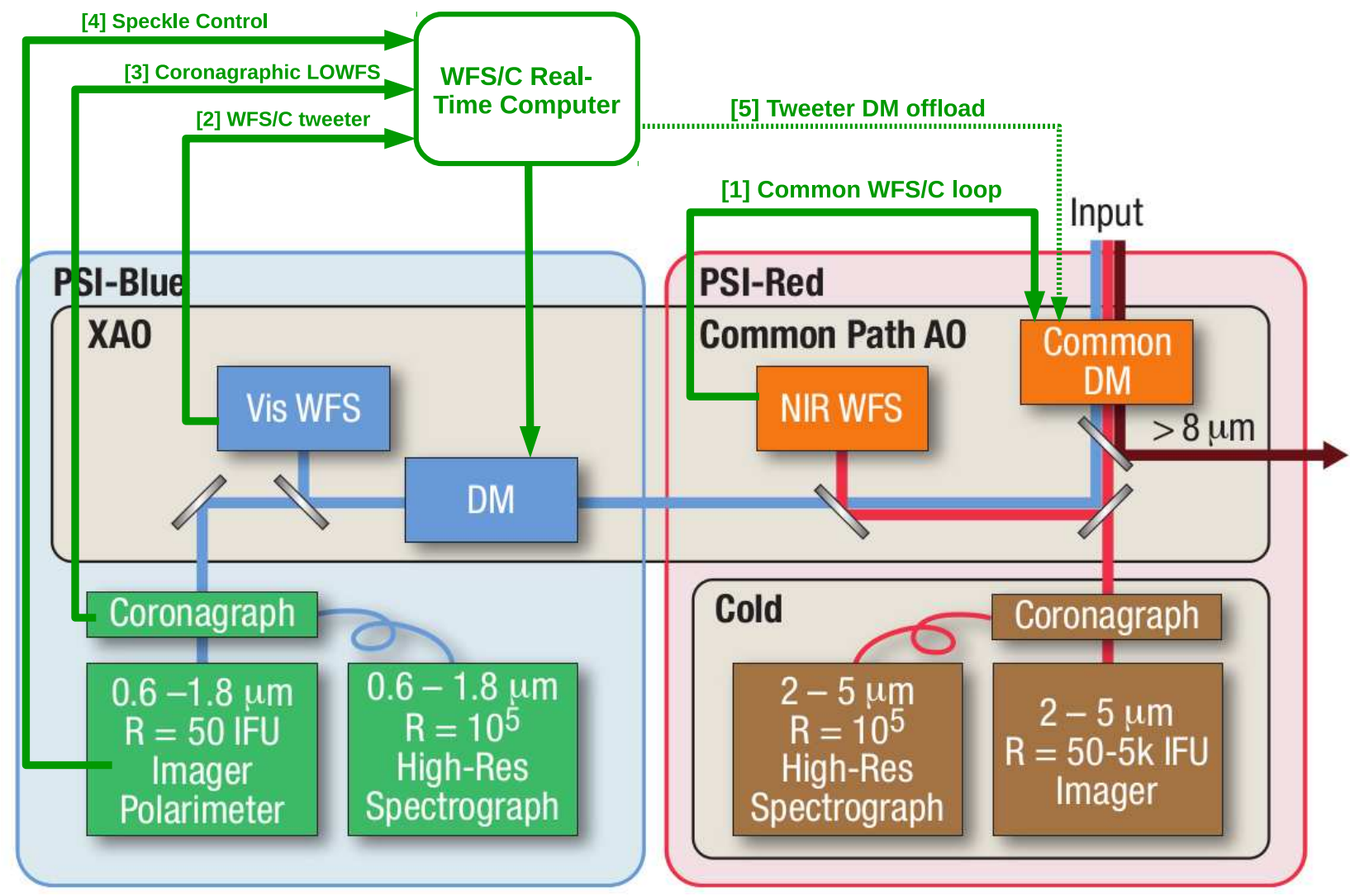

Figure 4. TMT PSI architecture. Wavefront control signal paths are shown in green.

\subsection{Control Algorithm}

While the PSI-red common WFS/C AO loop (loop [1]) can be a conventional stand-alone loop posing no particular implementation challenge, PSI-blue's WFS/C will employ advanced algorithms to meet the challenging $<1$ e- 5 raw contrast requirement:

- Sensor fusion will optimally combine measurements from the three WFS (ExAO visible WFS, coronagraphic LOWFS and focal plane speckle imaging) to control a single deformable mirror. Sensor fusion will require cross-calibration between the sensors, and a good understanding of each sensor's measurement null space.

- Active speckle control will use small wavefront modulations, added to PSI-blue's DM, to measure the complex amplitude of speckles in the focal plane. ${ }^{6,7}$ The measurements should be performed quickly (several $100 \mathrm{~Hz}$ ) to control atmospheric chromatic speckles described in §3.2: terms OMUL and ORPA in Figure 3. Focal plane speckle control, when performed over one half of the image, also addresses amplitude term UAMP.

- Coherent Differential Imaging will use DM modulations to measure both the coherent (starlight) and incoherent (planet light) components of the focal plane image. This technique requires a fast sensitive focal plane nearIR detector, such as MKIDs $^{8}$ or SAPHIRA. ${ }^{9}$

- Predictive control will eliminate temporal lag and allow more sensitive WFS measurements ${ }^{10,11}$ reducing terms OTEM and OPHN in Figure 3. The analytical model developed in $\S 3.2$ calls for $\approx 10 \mathrm{kHz}$ control 
loop frequency at low spatial frequencies, highlighting the need for low-lag control. Predictive control will deliver such low lag with significantly slower WFS frame rate.

These techniques are an active ongoing research area: their development and system integration is a large part of the overall development effort for PSI-blue, and will likely extend into the TMT-PSI instrument operation phase.

\section{CONCLUSIONS, ONGOING AND FUTURE WORK}

The TMT-PSI wavefront sensing and control requirements are imposed by direct imaging of exoplanets in reflected light. Using analytical models and fundamental signal-to-noise considerations, we have shown that a conceptual WFS/C architecture with two deformable mirrors and four WFSs does meet the challenging requirements, allowing spectroscopic characterization of habitable planets orbiting nearby M-type stars.

The WFS/C architecture is being validated on adaptive optics systems in operation and development on current large telescope. The Subaru Coronagraphic Extreme AO (SCExAO) instrument is validating many of the WFS/C solutions envisioned for TMT-PSI, and serves as a system-level prototyping platform. ${ }^{12,13}$ The MagAO$\mathrm{X}$ system, ${ }^{14}$ currently under development, also adopts similar WFS/C approaches. The KPIC instrument ${ }^{15}$ also validates the proposed near-IR wavefront sensing and fiber-based spectroscopy subsystems.

\section{ACKNOWLEDGMENTS}

The author acknowledges funding support from the Japanese Society for the Promotion of Sciences (JSPS), the Japanese Astrobiology Center, National Institutes for Natural Sciences (ABC/NINS). The authors acknowledge support from the JSPS (Grant-in-Aid for Research 23340051 and 26220704). The authors wish to acknowledge the Center for Adaptive Optics Fall Retreat and the TMT Science Forum, where much of this work originated.

\section{REFERENCES}

1. M. Fitzgerald, "The Planetary Systems imager: a high-contrast instrumentation platform for the Thirty Meter Telescope," Proc. SPIE 10702(74), 2018.

2. D. Mawet, "The Planetary Systems imager: 0.5-1.8 micron channel," Proc. SPIE 10702(310), 2018.

3. A. J. Skemer, D. Stelter, D. Mawet, M. Fitzgerald, M. Mazin, O. Guyon, C. Marois, Z. Briesemeister, T. Brandt, J. Chilcote, J.-R. Delorme, N. Jovanovic, J. Lu, N. Millar-Blanchaer, J. Wallace, G. Vasisht, L. Roberts, and J. Wang, "The Planetary Systems imager: 2-5 Micron Channel," Proc. SPIE 10702(371), 2018.

4. C. Marois, "Thermal Imaging of Earth-size temperate planets orbiting neighborhood stars," Proc. SPIE 10702(154), 2018.

5. O. Guyon, "Limits of Adaptive Optics for High-Contrast Imaging," ApJ 629, pp. 592-614, Aug. 2005.

6. F. Martinache, O. Guyon, N. Jovanovic, C. Clergeon, G. Singh, T. Kudo, T. Currie, C. Thalmann, M. McElwain, and M. Tamura, "On-Sky Speckle Nulling Demonstration at Small Angular Separation with SCExAO," PASP 126, p. 565, June 2014.

7. M. Bottom, J. K. Wallace, R. D. Bartos, J. C. Shelton, and E. Serabyn, "Speckle suppression and companion detection using coherent differential imaging," MNRAS 464, pp. 2937-2951, Jan. 2017.

8. S. R. Meeker, B. A. Mazin, R. Jensen-Clem, A. B. Walter, P. Szypryt, M. J. Strader, and C. Bockstiegel, "Design and Development Status of MKID Integral Field Spectrographs for High Contrast Imaging," in Adaptive Optics for Extremely Large Telescopes IV (AO4ELT4), p. E75, Dec. 2015.

9. D. E. Atkinson, D. N. B. Hall, I. M. Baker, S. B. Goebel, S. M. Jacobson, C. Lockhart, and E. A. Warmbier, "Next-generation performance of SAPHIRA HgCdTe APDs," in High Energy, Optical, and Infrared Detectors for Astronomy VII, Proc. SPIE 9915, p. 99150N, Aug. 2016.

10. O. Guyon and J. Males, "Adaptive Optics Predictive Control with Empirical Orthogonal Functions (EOFs)," ArXiv e-prints, July 2017.

11. J. R. Males and O. Guyon, "Ground-based adaptive optics coronagraphic performance under closed-loop predictive control," Journal of Astronomical Telescopes, Instruments, and Systems 4, p. 019001, Jan. 2018. 
12. A. Sahoo, O. Guyon, C. Clergeon, N. Skaf, Y. Minowa, and J. Lozi, "Subaru Coronagraphic Extreme-AO (SCExAO) wavefront control: current status and ongoing developments," Proc. SPIE 10703(187), 2018.

13. J. Lozi, O. Guyon, N. Jovanovic, S. Goebel, P. Pathak, N. Skaf, A. Sahoo, B. Norris, F. Martinache, M. NDiaye, B. Mazin, A. Walter, P. Tuthill, T. Kudo, H. Kawahara, T. Kotani, M. Ireland, N. Cvetojevic, E. Huby, S. Lacour, S. Vievard, T. D. Groff, J. K. Chilcote, J. Kasdin, J. Knight, F. Snik, D. Doelman, Y. Minowa, C. Clergeon, N. Takato, M. Tamura, T. Currie, H. Takami, and M. Hayashi, "SCExAO, an instrument with a dual purpose: perform cutting-edge science and develop new technologies," Proc. SPIE 10703(270), 2018.

14. J. R. Males, L. M. Close, K. Miller, L. Schatz, D. Doelman, J. Lumbres, F. Snik, A. Rodack, J. Knight, K. Van Gorkom, J. D. Long, A. Hedglen, M. Kautz, N. Jovanovic, K. Morzinski, O. Guyon, E. Douglas, K. B. Follette, J. Lozi, C. Bohlman, O. Durney, V. Gasho, P. Hinz, M. Ireland, M. Jean, C. Keller, M. Kenworthy, B. Mazin, J. Noenickx, D. Alfred, K. Perez, A. Sanchez, C. Sauve, A. Weinberger, and A. Conrad, "MagAO-X: project status and first laboratory results," Proc. SPIE 10703(9), 2018.

15. D. Mawet, C. Bond, J.-R. Delorme, N. Jovanovic, M. Chun, D. Hall, S. Cetre, S. Lilley, O. Guyon, J. Wallace, and P. Wizinovich, "Keck Planet Imager and Characterizer: status update," Proc. SPIE 10703(6), 2018. 